\title{
Atypical presentations of superficial dermatophytoses associated with Ruocco's immunocompromised cutaneous district: A case series
}

\author{
Ramesh Kumar Kushwaha', Alpana Mohta², Suresh Kumar Jain ${ }^{3}$
}

${ }^{1}$ Department of Dermatology, Venereology and Leprosy, Government Medical College, Kota, Rajasthan, India, ${ }^{2}$ Department of Dermatology, Venereology and Leprosy, Sardar Patel Medical College, Bikaner, Rajasthan, India

Corresponding author: Alpana Mohta, MD, E-mail: Dralpanamohta10@gmail.com

\begin{abstract}
An immunocompromised district is an area of irregular immune control of the skin occurring due to cutaneous damage of any sort conducive to the development of infections, immune reactions, and tumors. Superficial dermatophytoses are one of the most commonly encountered cutaneous infections, which, in some cases, may have various atypical presentations. Herein, we present a series of eleven such cases in which the presentation of a superficial dermatophytosis was altered by the concurrent presence of a different unrelated dermatosis on the same anatomical site.
\end{abstract}

Key words: Immunocompromised district; Locus minoris resistentiae; Superficial dermatophytosis; Atypical tinea

\section{INTRODUCTION}

In 2014, Ruocco defined an immunocompromised district (ICD) as areas of the skin with an immune microenvironment conducive to the development of a new disease [1]. An ICD is an area of irregular immune control of skin occurring due to cutaneous damage of any sort conducive to the development of infections, immune reactions, and tumors [2]. Lately, several case reports and series documented this privileged localization of one dermatosis over another $[2,3]$. In fact, the Koebner phenomenon is one such example.

Herein, we describe a case series of patients presenting with superficial dermatophytosis present on top of an unrelated dermatosis.

\section{CASES}

\section{Case 1}

A 32-year-old male, an under-treatment case of postherpetic neuralgia on the left side of the flank persistent for four months, presented with the development of annular, scaly pruritic plaques on the same dermatomes as the patient's past herpes zoster lesions from one month prior. A closer examination revealed the striking phenomenon of annular sparing seen around healed scars of herpes zoster. The patient was diagnosed with the development of tinea corporis on the healed lesions of herpes zoster.

\section{Case 2}

A 36-year-old male, a case of chronic plaque psoriasis persistent for three years, presented with a current exacerbation lasting for the past fifteen days. The patient was prescribed oral methotrexate with topical betamethasone lotion for the same condition in the past, but, for the last three months, was off all treatment. A cutaneous examination revealed, apart from silvery, salmon-colored, scaled and indurated plaques of psoriasis, widespread erythematous annular and polycyclic plaques on the existing lesions of psoriasis (Fig. 1). There was no history of preceding sore throat, fever, joint pain, or systemic involvement.

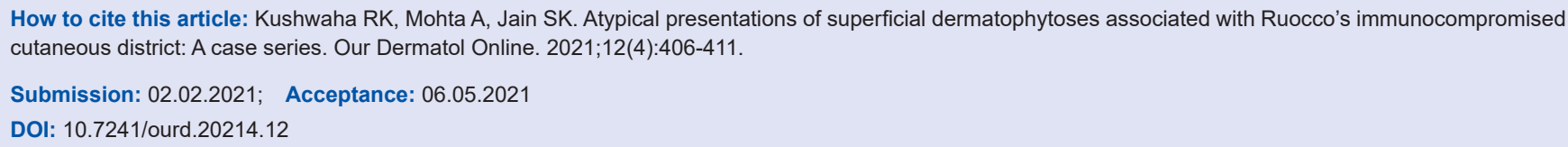


The patient was diagnosed with tinea corporis and tinea cruris with psoriasis.

\section{Case 3}

A known case of localized morphea, 30 years of age, presented with the development of an itchy, scaly lesion on an already existing lesion in the right subscapular region. An examination revealed well-defined erythematousto-hyperpigmented plaques with peripheral scaling. The background skin had an ill-defined, depressed plaque with a sclerodermatous appearance along with hyperpigmentation and a violaceous hue. The patient had been under treatment with $0.005 \%$ calcipotriol ointment for the last two months. The new scaly plaque had developed for the last one month. With a strong clinical suspicion, a scraping from the plaque was sent for $\mathrm{KOH}$ and fungal culture examination, both positive for dermatophytosis. A diagnosis of tinea incognito with morphea was reached. The patient was prescribed oral and topical antifungals, which led to the resolution of tinea incognito after four weeks (Fig. 2).

\section{Case 4}

A 50-year-old female, while under palliative radiotherapy for squamous cell carcinoma of the buccal mucosa, developed dermatophytic infection in the radiation port. The patient had been receiving radiotherapy for the last four months, while the lesions of tinea faciei had developed over the last one month, involving the left preauricular and mental area extending up to the angle of the mouth (Fig. 3). A diagnosis of radiationport dermatophytosis was reached confirmed by a positive fungal culture.

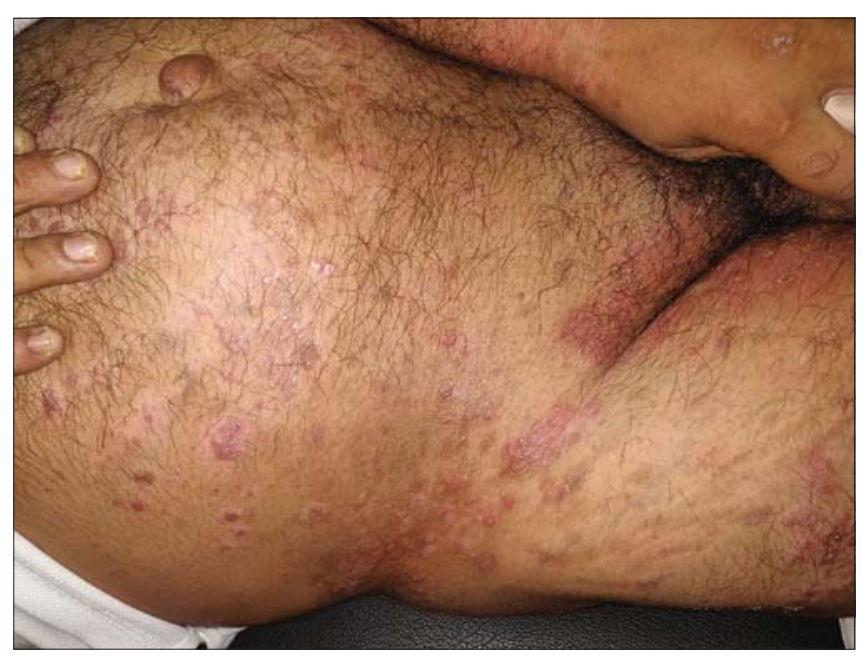

Figure 1: Round-to-oval plaques of psoriasis surmounted over tinea corporis on the abdomen.

\section{Case 5}

A 46-year-old male, a known, biopsy-proven case of dermatitis herpetiformis presented with the development of multiple annular, scaly and erythematous plaques over already existing lesions on the extensors, involving the elbows and buttocks. The patient had been taking tablet dapsone $100 \mathrm{mg}$ once a day for the same condition, along with a topical preparation of $0.05 \%$ betamethasone lotion. The new annular lesions had developed over the last one month. With a strong clinical suspicion, a $\mathrm{KOH}$ mount of the marginal scales was performed, confirming the diagnosis of tinea incognito.

\section{Case 6}

A 63-year-old male presented with a complaint of intense pruritus and scarring and darkening of the shin of the left leg persistent for six months, because of

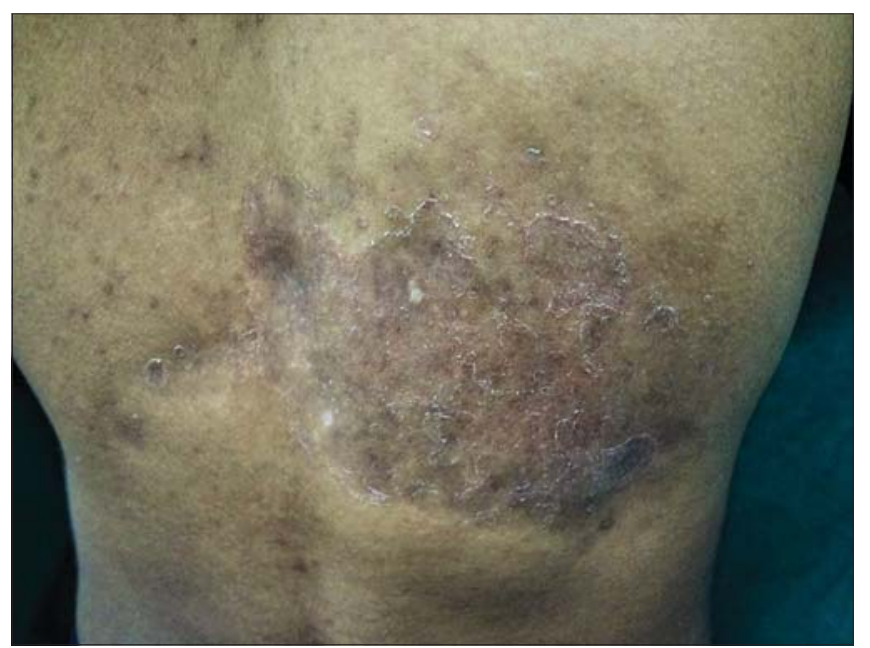

Figure 2: Tinea incognito on a localized morpheic plaque.

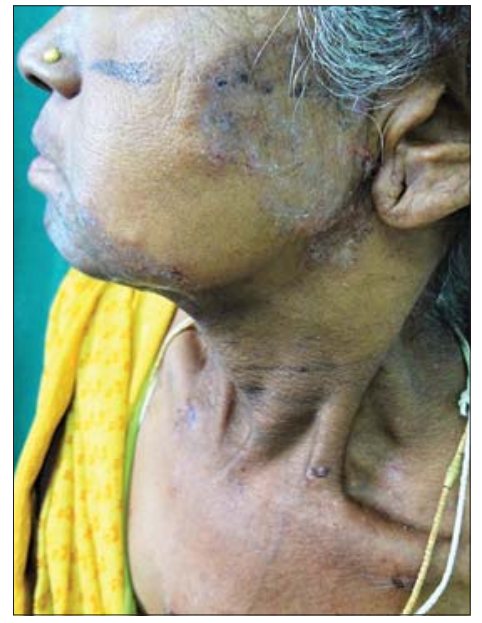

Figure 3: Radiation port dermatophytosis on the face. 
which he was diagnosed with lichen simplex chronicus three months earlier and for which he was prescribed liquid paraffin. Four months after the onset of these lesions, the patient developed a progressive, raised, annular erythematous plaque around the lesion, which had progressively increased in size and surrounded the entire lesion. The patient denied the use of any topical or oral steroid. A diagnosis of tinea corporis superimposed on lichen simplex chronicus was reached.

\section{Case 7}

A 56-year-old male, a laborer by profession, presented with complaints of itchy lesions on the trunk and neck. An examination revealed lesions consistent with tinea corporis. An incidental finding, however, was made during the examining and the presence of multiple depigmented vitiliginous macules within the plaques of tinea was noted. Surprisingly, all the vitiligo plaques were completely spared from evidence of superficial dermatophytosis, and there was a sharp demarcation between the islands of sparing and the surrounding scaly plaques of tinea corporis (Fig. 4). To confirm the sparing phenomenon, a scraping from the vitiliginous plaques was sent for a fungal culture, which was negative. A diagnosis of tinea corporis with a sparing phenomenon in vitiligo macules was reached.

\section{Case 8}

A 49-year-old male, a known case of tinea corporis with tinea cruris under treatment for the last six months, presented with the presence of an irregularly shaped, well-defined atrophic erythematous plaque on the right buttock (Fig. 5). The patient admitted that the plaque was slowly progressive and had been spreading in one direction while healing toward the other end. The lesion was biopsied and histopathology was found to be consistent with lupus vulgaris, showing epithelioid granuloma and pseudoepitheliomatous hyperplasia.

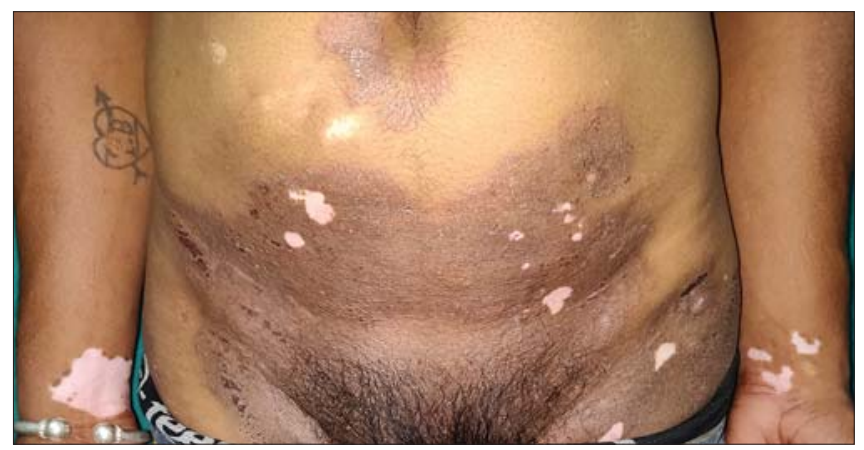

Figure 4: Islands of sparing in vitiligo macules within plaques of tinea corporis on the lower abdomen.
The patient also had a positive Mantoux test, but a chest X-ray and other routine investigations were unremarkable. The patient was put under treatment for cutaneous tuberculosis while the antifungal treatment was continued. The patient showed a marked improvement after three weeks of the treatment itself.

\section{Case 9}

A 36-year-old male presented with four days of history of high-grade fever along with an abrupt development of multiple intact or ruptured discreet vesicular lesions with crusting in some over the body. There was, however, a clustering of vesicles surmounted over well-defined, scaly erythematous plaques with raised borders on the right side of the face and in the groin area, which had been present for the last four months (Fig. 6). The patient had similar vesicular lesions over

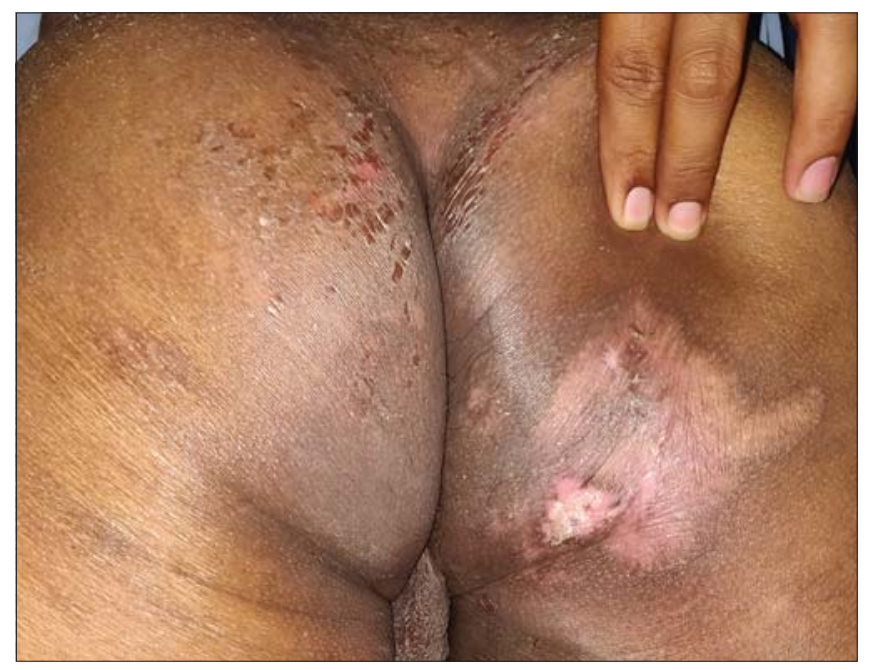

Figure 5: The presence of a plaque of lupus vulgaris within tinea corporis.

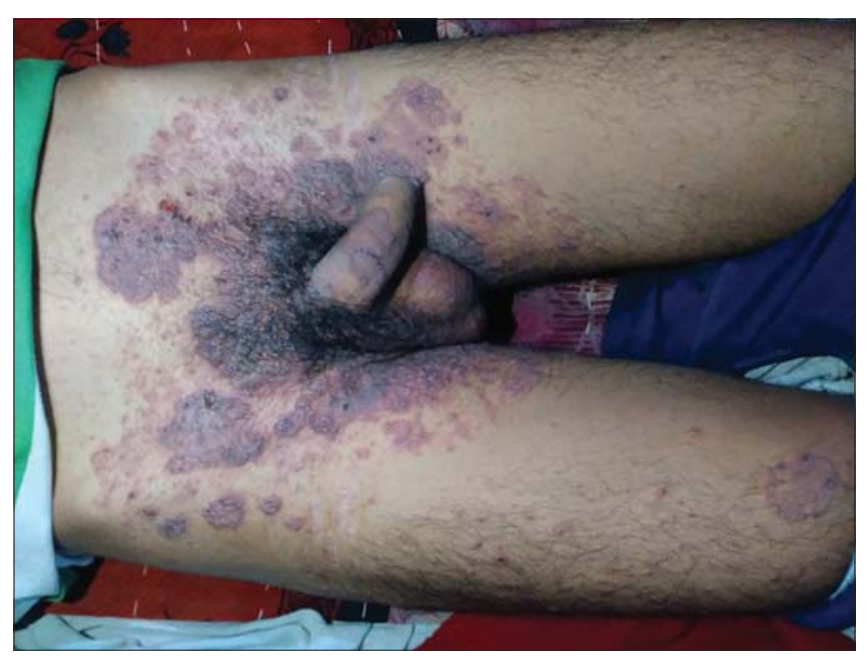

Figure 6: A clustering of varicella-zoster vesicles within plaques of tinea corporis. 
the entire body. The background plaque was diagnosed as tinea faciei with tinea cruris under high clinical suspicion, further confirmed by a $\mathrm{KOH}$ mount and fungal culture. The fluid collected from the base of the vesicular lesion was examined by a Tzanck smear, showing multinucleated giant cells. The patient was diagnosed with varicella-zoster with tinea faciei.

\section{Case 10}

Another case, a 43-year-old married, immunocompetent female patient, presented with complaints of itchy red lesions in the groin area. An examination revealed the presence of a well-defined polycyclic annular erythematous scaly plaque with multiple shiny white papules with umbilication in some. On inquiry, the patient said that this plaque had been present for six months, while the papules had developed in the last one month, and that her husband had similar papules on the genitalia. The patient also admitted to applying topical steroids to the lesion for the last two weeks. The patient was diagnosed clinically with molluscum contagious superimposed on tinea cruris, further confirmed by a KOH mount of the background plaque, while the shiny papules on extirpation demonstrated typical molluscum bodies.

\section{Case 11}

A 33-year-old female presented with similar complaints as case three in the groin area persistent for four months. The patient had already been an undertreatment case of tinea corporis for the last one and a half months. However, the new umbilicated papules had rapidly developed over the already existing plaque in the last three weeks. The patient's serology was negative for HIV, hepatitis B, and the VDRL test. Other routine investigations, including a hemogram and liver and renal function tests, were within normal limits. A diagnosis of molluscum contagiosum with tinea corporis was reached, further confirmed by the $\mathrm{KOH}$ test and extirpation.

\section{DISCUSSION}

This case series highlights the concept of immunocompromised cutaneous districts (ICD) in relation to superficial dermatophytosis $[1,2]$. Several factors contribute to ICDs, such as prolonged lymphatic stasis, viral infections such as herpes, radiation sources such as palliative radiotherapy, posttraumas, burns, etc., all leading to localized immune dysregulation. This phenomenon is also known as locus minoris resistentiae [2]. Such ICDs are vulnerable to the development of opportunistic fungal infections. The same was seen in cases one through six.

In case 1 , the development of tinea corporis over healing lesions of herpes zoster could be explained by three proposed hypotheses. According to the first hypothesis, the viral antigens and altered skin antigens lead to an atypical delayed hypersensitivity reaction or long-standing immunologic changes. Another hypothesis states that the neuropeptides, secreted from cutaneous nerves injured by the herpes virus, lead to the modulation of local immune and vascular proliferative responses. Lastly, according to the third hypothesis, herpes zoster induces anatomic changes such as scarring, the redistribution of collagen, microcirculation alteration, and defects in the skin barrier, deregulating the defense mechanism of the skin in the affected dermatome.

In case 4 , the diagnosis of radiation-port dermatophytosis (RPD) was reached. RPD is an elusive and sparsely reported entity. Its pathogenesis is still not fully understood, but this is an example of an ICD as well. Various studies in the past have demonstrated a significant reduction in the Langerhans cell count in mice even with a single dose of $20 \mathrm{~Gy}$. This reduction in immune surveillance coupled with a barrier dysfunction, fibrosis, and a sluggish blood flow contributes to the radiation port site becoming a privileged site for dermatophytosis. However, in all such cases, acute radiation dermatitis must be excluded first [4].

The same concept of immune dysregulation due to chronic itching altering the lymphatic and immune regulation could explain the development of dermatophytosis within a plaque of lichen simplex chronicus in case 5.

In search through the literature of the incidence of dermatophytic infections on psoriatic lesions for case 2, we found that such a combination is a very rarely reported entity, for both keratinophilic and keratinolytic dermatophytes. The parakeratotic cells of psoriasis are unfavorable for the development of such fungal infection. Although several reports in the past have mentioned the development of onychomycosis in psoriatic nails, the presence of superficial dermatophytosis superimposed on psoriatic plaques is an under-reported entity. 
Case 3 was a unique case of tinea incognito secondary to topical calcipotriol application. Although several cases of the development of tinea incognito secondary to an erroneous topical administration of corticosteroids and calcineurin inhibitors have been described, to the best of our knowledge, none so far have occurred secondary to the sole application of calcium channel analogs. The application of immunosuppressive or immunomodulatory drugs leads to the downregulation of local immunity against fungi by suppression of beta-defensins and T-cell-mediated immunity. This phenomenon could be partly classified as a pharmacotopic response, secondary to calcipotriol, contributing to the development of an ICD [5-8].

The sparing of vitiligo and contact leukoderma lesions in cases of parthenium dermatitis and textile dermatitis has been described. There are also reports of sparing of nevus depigmentosus in cases of generalized drug eruption. The underlying pathomechanism included the vacuolization of Langerhans cells in these depigmented areas. However, no reports so far on the sparing of vitiligenous macules in dermatophytosis have been published.

There have been reports of lupus vulgaris masquerading as superficial dermatophytosis. The buttocks are a commonly misdiagnosed site involving lupus vulgaris due to its rarity or sporadic presentation in atypical forms [9].

The clearance of a dermatophytic infection is attributed to the upregulation of cell-mediated immunity dependent on $T_{h} 1 / T_{h} 17$. However, the concurrent presence of chronic skin diseases such as untreated cutaneous tuberculosis, psoriasis, atopic dermatitis, and dermatitis herpetiformis (as seen in case 5) shifts the immune response to the $\mathrm{T}_{\mathrm{h}} 2$ spectrum making the host site more susceptible to superficial mycosis [10]. Irrespective of the underlying cause, the precise mechanism leading to this skewing of the immune response and the absence of a cell-mediated immune response to dermatophytic invasion is still unclear. The aberrant antigen processing of the fungal hyphae and phagocytosis by the polymorph nuclear leukocytes and macrophages along with hypocomplementemia and Caspase recruitment domain-containing protein 9 (CARD9) gene mutation may be contributing factors [10].

The localization of varicella and, by extrapolation, other cutaneous viral infections such as poxvirus infection (molluscum contagiosum) on the sites of active dermatophytosis could be attributed to the presence of various migration pathways of cutaneous lymphocyte antigen memory T cells involved in immune surveillance. In the case of active inflammation, the migration of these $\mathrm{T}$ cells to the skin is significantly increased. Interestingly, the varicella-zoster virus has a high affinity for infecting these cells. Once these infected T cells localize to the site of active inflammation (e.g., superficial dermatophytosis), there is a clinically visible exuberant growth of these diseases over sites of tinea, as seen in cases 1 and 2. The same concept may also be applied to the acquisition of molluscum contagiosum over such sites of dermatophytic infection [11].

\section{CONCLUSION}

Our series highlights the importance of diagnosing various atypical presentations of superficial dermatophytoses with respect to the presence of other superimposed dermatoses. The concurrent occurrence of two unrelated dermatoses in the same sector of an immunocompromised district of the skin is both a diagnostic and therapeutic challenge for a dermatologist and one must always be on the lookout for such atypical cases in case of chronic and recurrent dermatophytoses. Antifungal resistant cases are an everrising phenomenon globally. An erroneous judgment in diagnosing such cases may add to this uprise of resistant fungal infections. Our series may be a useful tool for studies to come in the future aimed at exploring the same subject matter.

\section{Consent}

The examination of the patient was conducted according to the principles of the Declaration of Helsinki.

The authors certify that they have obtained all appropriate patient consent forms, in which the patients gave their consent for images and other clinical information to be included in the journal. The patients understand that their names and initials will not be published and due effort will be made to conceal their identity, but that anonymity cannot be guaranteed.

\section{REFERENCES}

1. Ruocco V, Ruocco E, Piccolo V, Brunetti G, Guerrera LP, Wolf R. The immunocompromised district in dermatology: A unifying pathogenic view of the regional immune dysregulation. Clin Dermatol. 2014;32:569-76.

2. Ranugha P, Betkerur JB, Veeranna S, Basavaraj V. Appearance of verruca over linear verrucous epidermal nevus - An example of locus minoris resistentiae: A report of three cases. Indian Dermatol 


\section{www.odermatol.com}

Online J. 2018;9:334-7.

3. Long R, Beatch A, Lee MC, Cheung Lee M, Wasel N. Lupus vulgaris occurring in a locus minoris resistentiae. J Cutan Med Surg. 2009;13:313 6 .

4. Cheng YP, Sun CC, Liao YH. Diagnosis and treatment of radiation port dermatophytosis of scalp: A case report. Mycoses. 2012;55:e27-8.

5. Starace M, Alessandrini A, Piraccini BM. Tinea incognita following the use of an antipsoriatic gel. Skin Appendage Disord. 2016;1:123-5

6. Segal D, Wells MM, Rahalkar A, Joseph M, Mrkobrada M. A case of tinea incognito. Dermatol Online J. 2013;19:18175.

7. Rallis E, Koumantaki-Mathioudaki E. Pimecrolimus induced tinea incognito masquerading as intertriginous psoriasis. Mycoses. 2008;51:71-3.
8. Verma S, Hay RJ. Topical steroid-induced tinea pseudoimbricata: A striking form of tinea incognito. Int J Dermatol. 2015;54:e192-3.

9. Heo YS, Shin WW, Kim YJ, Song HJ, Oh CH. Annular lupus vulgaris mimicking tinea cruris. Ann Dermatol. 2010;22:226-8.

10. Dogra S, Narang T. Emerging atypical and unusual presentations of dermatophytosis in India. Clin Dermatol Rev. 2017;1:Suppl S1-12.

11. Yi JS, Cox MA, Zajac AJ. T-cell exhaustion: Characteristics, causes and conversion: T-cell exhaustion. Immunology. 2010;129:474-81.

Copyright by Ramesh Kumar Kushwaha, et al. This is an open access article distributed under the terms of the Creative Commons Attribution License, which permits unrestricted use, distribution, and reproduction in any medium, provided the original author and source are credited.

Source of Support: Nil, Conflict of Interest: None declared. 